\title{
Sinopse de Artigos Mais Relevantes em Arritmias Cardíacas
}

\author{
Synopsis of Most Relevant Articles on Cardiac Arrhythmias
}

\author{
Editor Associado: Bruno Papelbaum \\ Papelbaum B (iD https://orcid.org/0000-0001-7154-7001 \\ 1.Centro Avançado de Ritmologia e Eletrofisiologia - São Paulo (SP) - Brasil. \\ E-mail: brpapel@gmail.com
}

\section{Incompetência Cronotrópica com Preditor de Risco em Crianças e Adultos Jovens Com Taquicardia Ventricular Polimórfica Catecolaminérgica*}

Taquicardia ventricular polimórfica catecolaminérgica (TVPC) é uma canalopatia hereditária potencialmente letal. Dados recentes sugerem uma história natural desfavorável, com quase $80 \%$ dos pacientes sofrendo um evento cardíaco ameaçador à vida próximo dos 40 anos. O teste ergométrico (TE) traz informações sobre ectopias ventriculares (EV) e sua densidade como preditor de eventos cardíacos; a incompetência cronotrópica (IC) ou frequência cardíaca (FC) atenuada também são prognósticos em outras condições. Os autores propõem, portanto, que a IC durante o exercício pode predizer uma incidência arrítmica maior em pacientes com TVPC. O estudo foi uma análise retrospectiva de pacientes jovens ( $\leq 21$ anos) com TVPC, em centros terciários como o British Columbia Children's Hospital e o Monroe Carell Jr. Children's Hospital em Vanderbilt. Dois TEs por paciente foram analisados (14 sem terapia e 17 em terapia máxima), com monitoramento da FC e pressão arterial (PA); o esforço máximo atingido foi medido em equivalentes metabólicos (METS) e tempo total de exercício (em segundos). O escore de arritmias ventriculares (EAV) foi determinado de acordo com: $\operatorname{sem} \mathrm{EV}=0$, batimentos ventriculares prematuros isolados $=1$, bigeminismo $=2$, pares $=3$, e taquicardia ventricular não sustentada $=4$. A idade média ao primeiro TE foi de 12 anos, com 45\% pacientes do sexo masculino, sendo a síncope relatada em seis pacientes (30\%) e morte súbita cardíaca (MSC) em oito pacientes (40\%), enquanto os seis restantes (30\%) eram assintomáticos e foram submetidos a rastreio genético familiar. As causas para interromper o TE no grupo sem medicação foram fadiga (40\%), arritmia cardíaca, TV bidirecional em 40\%, e alvo de FC atingida em 20\%; 5 de 14 pacientes sem terapia apresentaram IC (36\%). Os pacientes com IC foram os que apresentaram pior EAV quando comparados aos pacientes com cronotropismo normal $(3,4 \pm 0,6$ vs $1,4 \pm 0,6 ; P=0,046)$, menor pressão arterial (PA) diastólica de repouso ( $65 \pm 3$ vs $74 \pm 2 ; P=0,045)$, menor PA de pico sistólica $(133 \pm 10$ vs $168 \pm 10 ; P=0,041)$ e menor delta de PA sistólica (pico PA sistólica menos PA sistólica de repouso; $24 \pm 8$ vs $59 \pm 11 ; P=0,047$ ). Dos pacientes com medicação, 10 de 17 (59\%) tiveram IC (reserva de FC $\leq 62 \%$ ); as causas para interromper o TE foram fadiga em $80 \%$, falta de ar em 10\% e ansiedade em outros 10\%. Um detalhe importante sobre o TE foi que ele era interrompido/ limitado por sintoma/arritmia e não FC alvo. Não houve diferença no EAV entre aqueles com IC e cronotropismo normal em terapia máxima $(P=0,05)$. Esses pacientes com IC em terapia máxima continuaram a apresentar menor pico de PA sistólica (126 $\pm 6,2$ vs 151,4 $\pm 9,5 ; P=0,033)$ comparados aos pacientes sem IC. Não houve associação entre idade, duração de exercício, METS atingidos, FC em repouso, PA sistólica em repouso, PA diastólica em repouso e delta de PA sistólica. Terapia com beta-bloqueador apresentou um efeito depressor no pico da FC ao esforço em pacientes com 
IC (sem terapia: $161,2 \pm 5,4$ vs terapia máxima: $128,5 \pm 6,4 ; P=0,0058$ ) e cronotropismo normal (sem terapia: 197,8 $\pm 3,3$ vs terapia máxima: $157,1 \pm 3,4 ; P<0,0001)$. Nove de dez pacientes $(90 \%)$ com TVPC e IC em terapia máxima necessitaram de terapia adicional além do beta-bloqueador, comparados a $43 \%$ com cronotropismo normal $(P=0,042)$, sem diferenças quanto a implante de cardiodesfibrilador. Os autores concluem que o TE promove informações clínicas valiosas além do diagnóstico de TVPC, sendo a IC um marcador de risco para TVPC, que parece ser atenuada com terapia antiarrítmica. Uma resposta relativamente hipotensora ao exercício pode ser um novo marcador prognóstico. Os achados do estudo implicam que o sistema nervoso autônomo tem papel na modulação da doença.

*Franciosi S, Roston TM, Perry FKG, Knollmann BC, Kannankeril PJ, Sanatani S. Chronotropic incompetence as a risk predictor in children and young adults with catecholaminergic polymorphic ventricular tachycardia. J Cardiovasc Electrophysiol. 2019;30(10):1923-9. https://doi.org/10.1111/jce.14043

\section{Efeitos dos Anticoagulantes de Ação Direta, Warfarina e Agentes Antiplaquetários no Risco de Hematoma de Loja. Análise Combinada do Bruise Control 1 e $2^{*}$}

As incidências de hematoma de loja variam muito: 1,2\% quando não há anticoagulante em uso, de 2,3 a 6,5\% com terapia continuada de warfarina e de 7 a 16\% durante ponte com heparina. O estudo Bruise Control 1 (BC1) demonstrou um aumento em mais de sete vezes no risco de infecção do dispositivo em hematomas clinicamente significantes (HCS), que necessitam de reoperação ou resultam em maior tempo de hospitalização; se a reintervenção for necessária, a chance de infecção aumenta em mais de 15 vezes. O BC1 demonstrou 80\% menos hematoma de loja quando a cirurgia foi realizada sem interrupção de warfarina comparado à estratégia de ponte com heparina (3,5 e 16\% respectivamente). O Bruise Control 2 (BC2) avaliou o uso de anticoagulantes de ação direta (DOACs, do inglês direct oral anticoagulants), sendo interrompido precocemente devido a não diferença em HCS entre o braço continuado de DOAC (2,1\%; 95\% IC, 0,9-4,3\%) e o braço interrompendo $\mathrm{DOAC}(2,1 \%$; 95\% IC, $0,9-4,3 \% ; P=0,97)$. O estudo em questão avaliou dados do $\mathrm{BC} 1$ e $\mathrm{BC} 2$ e efeitos do uso concomitante de antiagregantes em HCS, e visou o entendimento do risco de hematoma de ferida em pacientes tratados com DOAC versus warfarina continuada; ambos os estudos foram multicêntricos, randomizados, simples-cegos. O estudo incluiu 1.343 pacientes: 681 do $\mathrm{BC} 1$ e 662 do $\mathrm{BC} 2$. $\mathrm{BC} 1$ arrolou pacientes com risco preditivo anual de tromboembolismo de $5 \%$ ou mais, em uso de warfarina e planejando implante eletivo de dispositivo; o BC2 arrolou pacientes com fibrilação atrial não reumática e $\mathrm{CHA}_{2} \mathrm{DS}_{2}-\mathrm{VASc} \geq 2$ tratados com DOAC e com implantes eletivos. Dos 1.343 pacientes incluídos, $408(30,4 \%)$ foram identificados como utilizando ao menos um antiagregante no momento da cirurgia e 935 pacientes $(69,6 \%)$ não utilizavam. Daqueles, 383/408 (93,9\%) faziam uso de aspirina, 50/408 (12,3\%) de clopidogrel e 25/408 (6,1\%) de ambos. O desfecho primário de HCS ocorreu em 40/408 (9,8\%) pacientes com antiagregante versus 40/935 (4,3\%) no grupo sem antiagregante $(P<0,001)$, gerando mais reoperação $(2 \%$ vs $0,6 \%)$ e necessidade de interromper anticoagulação oral (9,1\% vs 3,9\%); em pacientes com terapia antiagregante dupla a ocorrência de HCS foi de 2/25 (8\%) comparado a 38/383 (9,9\%) em uso de um antiagregante $(P=1,0)$. Em análise multivariada, não houve diferença em HCS entre DOAC interrompido ou continuado versus warfarina continuada (Razão de chance, RC, 0,858; 95\% IC, 0,375-1.963; $P=0,7174$ ). $\mathrm{O}$ uso de antiagregante em adição a DOAC interrompido ou continuado foi fator preditor de HCS (RC, 1,965; 95\% IC, 1,202-3,213; $P=0,0071)$. Os autores concluem que, nos pacientes combinados dos estudos $\mathrm{BC} 1 \mathrm{e} \mathrm{BC2}$, o uso concomitante de terapia antiagregante dobrou o risco de HCS e a interrupção de antiagregante como parte da estratégia perioperatória deve ser considerada com cautela.

\footnotetext{
*Essebag V, Healey JS, Joza J, Nery PB, Kalfon E, Leiria TLL, et al. Effect of Direct Oral Anticoagulants, Warfarin, and Antiplatelet Agents on Risk of Device Pocket Hematoma. Combined Analysis of BRUISE CONTROL 1 and 2. Circ Arrhythm Electrophysiol. 2019;12(10):1-8. https://doi.org/10.1161/CIRCEP.119.007545
} 


\section{Cardioversão Elétrica de Fibrilação Atrial em Pacientes com Dispositivos de Oclusão do Apêndice Atrial Esquerdo*}

Cardioversão elétrica externa (CVE) é uma estratégia comum para controle de ritmo em fibrilação atrial (FA) e flutter atrial (FLA) de pacientes sintomáticos, tendo associação com risco pequeno de AVC ou embolia sistêmica, ficando em torno de 0,3 a 1\%. Na ausência de anticoagulação, o risco dessas complicações aumenta em mais de duas vezes. O uso de dispositivos de oclusão do apêndice atrial esquerdo (OAAE) surgiu como alternativa para prevenção de embolização sistêmica em pacientes com FA não candidatos ao uso prolongado de anticoagulante oral (ACO); nesses pacientes, não há dados de longa data relacionados à segurança e viabilidade de CVE após o implante de OAAE. Este foi um estudo retrospectivo, multicêntrico, envolvendo pacientes consecutivos em FA/FLA com OAAE pelo dispositivo Watchman (Boston Scientific, Marlborough, Massachusetts) submetidos a CVE eletiva entre julho de 2013 e julho de 2017. Os desfechos clínicos incluíram segurança e viabilidade de se realizar CVE após implante de OAAE. Todos os pacientes receberam regime com antitrombóticos incluindo seis semanas de aspirina $81 \mathrm{mg}$ ao dia com warfarina (INR entre 2-3)/ACO de ação direta (DOAC); os pacientes migraram para aspirina $81 \mathrm{mg}$ e clopidogrel $75 \mathrm{mg}$ ao dia após novo ecocardiograma transesofágico (ECO TE) ou tomografia computadorizada (TC), demonstrando posição satisfatória do dispositivo, sem trombo relacionado ao dispositivo (TRD) ou vazamento ao redor do dispositivo $\leq 5 \mathrm{~mm}$. Na sequência, os pacientes iniciavam somente aspirina $81 \mathrm{mg}$ após seis meses e novo ECO TE ou TC. Com relação à CVE, todos os pacientes foram submetidos a novo ECO TE logo antes dela e, caso algumas das condições anteriormente citadas não estivesse de acordo, a CVE não era realizada e os pacientes mantinham ACO por mais 4-6 semanas com novo exame de revisão. Foram arrolados 148 pacientes, com idade média de $72 \pm 7$ anos, 59\% do sexo masculino e $49 \%$ com FA paroxística; o $\mathrm{CHA}_{2} \mathrm{DS}_{2}$-VASC e HAS-BLED médios foram de 3,8 $\pm 1,7$ e 3,4 $\pm 1,6$, respectivamente. No momento da CVE, 34\% (51 de 148) estavam com ACO, 30\% (44 de 148) com terapia antiagregante dupla e 36\% (53 de 148) somente com aspirina $81 \mathrm{mg}$ ao dia. Imediatamente após a CVE, $22 \%$ (32 de 148) reiniciaram ACO com duração média de uso de 12 semanas após a CVE, e os pacientes com uso de ACO foram submetidos a CVE mais cedo do que os que estavam com antiagregante (3,6 meses [0,7 a 8,6 meses] versus 8,6 meses [2,5 a 13,3 meses]; $P=0,003)$. Não houve complicação tromboembólica relacionada à CVE, mas três ataques isquêmicos transitórios após 3,5 meses da CVE. Ocorreram 4\% (6 de 148) de sangramentos, quatro maiores (fatais ou com queda de hemoglobina $>3 \mathrm{~g} / \mathrm{dL}$ ) e dois menores; mais eventos ocorreram em pacientes mantidos com ACO, mas sem diferença estatisticamente significativa. Os autores concluem que os resultados preliminares de $\mathrm{CVE}$ em pacientes de alto risco com FA e após OAAE sem a necessidade de ACO se o ECO TE evidenciasse boa posição do dispositivo, ausência de TRD e vazamento $<5 \mathrm{~mm}$, são favoráveis, contudo maiores estudos devem ser realizados.

\section{Ablação por Radiofrequência versus Crioablação no Tratamento da Fibrilação Atrial Paroxística: Uma Metanálise*}

Fibrilação atrial (FA) é uma arritmia comum que afeta até $2 \%$ da população, atinge comumente idosos, com $26 \%$ de risco em homens e 23\% de risco em mulheres aos 80 anos. A primeira linha no tratamento da FA inclui agentes farmacológicos, que focam em controle da frequência cardíaca ( $\mathrm{FC}$ ), controle do ritmo e anticoagulação oral (ACO). Em pacientes com FA paroxística refratários a droga, a ablação por cateter com isolamento das veias pulmonares é a terapia-padrão; isso pode ser feito com radiofrequência (RF), com lesão por calor, ou com crioablação (CRIO), isto é, lesão por congelamento. RF é considerada a terapia-padrão e é mais utilizada do que a CRIO, entretanto, é mais longa e requer treinamento extenso, enquanto a CRIO possui efetividade similar à RF com menores taxas de complicação. Atualmente, está em debate qual o melhor tratamento para ablação em FA; metanálises prévias foram feitas, mas resultaram em inconclusão ou incluíram um 
número grande de desenhos de estudo, limitando a qualidade geral e a validade externa. O objetivo do estudo foi comparar a efetividade de longa data medida por sobrevida livre de FA em 12 meses de seguimento e complicações relacionadas aos dois métodos. Todos os estudos alocaram randomicamente pacientes $\geq 18$ anos tratados com RF ou CRIO, sendo excluídos estudos não randomizados. Os desfechos primários foram sobrevida livre de FA em 12 meses de seguimento e taxas de complicação; os desfechos secundários foram tempo de procedimento, de radioscopia e de ablação. Um total de 247 artigos foi identificado pela pesquisa sistemática na literatura; após um screening inicial de título e resumo, 17 artigos foram considerados e recuperados para se avaliar sua elegibilidade através de revisão do texto completo. Destes, um total de oito satisfizeram os critérios pré-especificados de inclusão e foram incluídos na revisão, incluindo 1.548 pacientes submetidos a ablação por RF ou CRIO. Todos foram publicados entre 2012 e 2015 e foram conduzidos em centros na Europa continental, no Reino Unido e na Rússia. Sete compararam CRIO com RF utilizando cateter irrigado, enquanto um comparou CRIO com RF do tipo faseada (phased duty-cycled). Com relação à sobrevida livre de FA, dos pacientes tratados, 53\% (393/741) submetidos a ablação com CRIO e 53\% (399/751) submetidos a RF desenvolveram FA em período $\geq 12$ meses, sem diferenças entre o tipo de energia utilizada (razão de chance, $\mathrm{RC}=0,98$, índice de confiança, IC = 0,67-1,43, $P=0,9)$ e, mesmo retirando o estudo com cateter faseado, não houve diferença ainda assim nas energias utilizadas. Com relação ao tempo de procedimento, no geral, a CRIO foi mais rápida em 4,08 minutos, entretanto essa diferença não atingiu significância estatística. Seis estudos avaliaram o tempo de radioscopia $(n=1.204)$, sendo 33,11 minutos com CRIO e 31,94 minutos com RF, também sem atingir significância estatística (diferença das médias, $\mathrm{DM}=1,17, \mathrm{IC}=-4,94-7,28$, $\left.I^{2}=87 \%, P=0,71\right)$. Dois estudos avaliaram o tempo de ablação $(n=155)$, sendo 99,02 minutos com CRIO e 91,06 minutos com $\mathrm{RF}$, também sem atingir significância estatística ( $\left.\mathrm{DM}=7,97, \mathrm{IC}=-35,15-51,09, I^{2}=95 \%, P=0,72\right)$. Sete estudos analisaram complicações pós-operatórias com as duas fontes de energia $(n=1.492)$; a despeito do tipo de complicação e de sua natureza transitória, a CRIO apresentou 1,9 vez mais chance de eventos quando comparada à RF, entretanto essa diferença não atingiu significância estatística e os dados se mantiveram inalterados após análise de sensibilidade. Lesão do nervo frênico teve 10,3 vezes mais chance de ocorrer com CRIO e cinco estudos relataram ser transitória e com resolução em 12 meses, sendo permanente em um estudo e em um paciente. Quanto ao tamponamento cardíaco, não houve diferenças entre os procedimentos $\left(\mathrm{RC}=0,39, \mathrm{IC}=0,11-1,40, I^{2}=0 \%, P=0,15\right)$. Os autores concluem que os resultados dessa metanálise demonstram taxas de sucesso global similares em 12 meses, com taxas comparáveis de radioscopia, tempos de procedimento e complicações em longo prazo entre CRIO e RF. Baseado no resultado, os operadores devem escolher a tecnologia com base nas características dos pacientes, suas preferências, bem como experiência e preferência do operador.

*Hachem AH, Marine JE, Tahboub HA, Kamdar S, Kanjwal S, Soni R, Kanjwal K. Radiofrequency Ablation versus Cryoablation in the Treatment of Paroxysmal Atrial Fibrillation: A Meta-Analysis. Cardiol Res Pract. 2018:1-10. https://doi.org/10.1155/2018/6276241

\section{Risco de Mortalidade Após Ablação por Cateter de Fibrilação Atrial*}

Ablação por cateter (AC) já está estabelecida como importante tratamento da fibrilação atrial (FA) sintomática, levando à melhora significativa na qualidade de vida dos pacientes. Além do mais, em pacientes com insuficiência cardíaca (IC) sistólica, a ablação se mostrou superior à terapia farmacológica em redução de mortalidade global e hospitalização por IC congestiva (ICC). Como os procedimentos vêm aumentando gradativamente, há a necessidade de se entender as taxas de complicações graves no mundo real, e estudos recentes sugerem uma maior tendência de complicações relacionadas à ablação a despeito de avanços nas tecnologias de cateteres e experiência do operador. Até o presente, as taxas de morte intrahospitalares variam de 0 a $0,8 \%$, entretanto estes dados são relacionados a centros acadêmicos, banco de dados regionais, de sistemas de saúde de pagador único e nacionais restritos às admissões para ablação. Já que podem ocorrer complicações após ablação como lesão esofágica, sepse e ICC, os autores levantaram a hipótese de que uma proporção de eventos ocorreria durante a readmissão e não durante a admissão para o procedimento e, utilizando um banco de dados nacional representativo, procuraram promover evidências do mundo real nas taxas, tendências e preditores de mortalidade precoce após ablação de 
FA, definida por mortalidade intra-hospitalar combinada da internação inicial (para o procedimento) ou readmissão 30 dias após o procedimento. Todos os dados foram obtidos da agência norte-americana para pesquisa e qualidade em saúde (Agency for Healthcare Research in Quality - AHRQ) de 2010 a 2015. O volume anual de ablação para FA foi determinado em uma base anual, calculando o número total de procedimentos realizados em uma instituição particular em determinado ano. Os desfechos primários foram mortalidade global precoce após ablação para FA, definida como mortalidade ocorrendo na internação inicial ou na readmissão 30 dias após o procedimento. Outros desfechos incluíram perfuração/tamponamento, outras complicações cardíacas iatrogênicas, complicações do sistema nervoso central, complicações vasculares e pneumotórax. Um total de 60.203 admissões foram registradas; a taxa global de mortalidade precoce após ablação foi de 0,46\% (95\% intervalo de confiança, IC: 0,37 a 0,52\%). Dos 276 óbitos precoces, 126 (45,7\%) ocorreram na internação inicial e 150 (54,3\%) na readmissão; comparados aos sobreviventes, os pacientes que faleceram eram mais idosos e tinha maiores comorbidades com ICC, doença arterial coronariana, implante prévio de marcapasso, hipertensão pulmonar, doença pulmonar crônica, doença renal crônica, anemia e coagulopatia (40,1\% versus 14,4\% com escore de Elixhauser de comorbidade $\geq 4 ; P<0,001$ ). Os pacientes que faleceram precocemente tiveram seus procedimentos realizados em centros de menor volume, residiam em áreas de menor renda e tiveram períodos mais longos de internação. A taxa global de qualquer complicação relacionada ao procedimento foi de 6,7\% e os pacientes que faleceram precocemente tiveram maiores complicações relacionadas ao procedimento (25,6 versus $6,6 \% ; P<0,001)$, com mais perfuração cardíaca, outras complicações cardíacas, complicações neurológicas e pneumotórax. Com relação a preditores de mortalidade precoce após ablação de FA, após ajuste para idade, comorbidades e características hospitalares, as complicações do procedimento foram independentemente associadas com mortalidade precoce (razão de chance ajustada, RCa: 4,06; IC = 95\%: 2,40 a 6,85; $P<0,001$ ), além de ICC (RCa: 2,20; $\mathrm{IC}=95 \%: 1,20$ a 4,03; $P=0,011)$, anemia (RCa: 1,83; IC = 95\%: 1,13 a 2,96; $P=0,015)$, coagulopatia (RCa: 2,14; $\mathrm{IC}=95 \%: 1,04$ a 4,39; $P=0,046)$ e idade (RCa: 1,04; IC = 95\%: 1,00 a 1,07; $P=0,046)$. Finalmente, pacientes submetidos a ablação em centros de baixo volume apresentaram maiores chances de mortalidade precoce (RCa: 2,35; IC = 95\%: 1,33 a 4,15; $P=0,003)$, sendo nesses casos os maiores preditores independentes: perfuração cardíaca, outras complicações cardíacas e complicações neurológicas como ataque isquêmico transitório/AVC. As causas de readmissão nos 150 pacientes com óbito foram cardíacas (30\%), infecciosas (30\%), respiratórias (17\%) e neurológicas (12\%). Os quatro diagnósticos mais comuns foram septicemia (15\%), ICC (15\%), pneumonia (7,4\%) e AVC $(5,9 \%)$ e os pacientes foram submetidos mais comumente a ventilação mecânica, transfusão sanguínea, ecocardiograma, endoscopia, diálise, instalação de tubo torácico, cateterização cardíaca direita, broncoscopia, suporte circulatório mecânico e toracocentese. A taxa de $0,46 \%$ encontrada no presente estudo excede as taxas de mortalidade relacionadas ao procedimento reportadas por outros grandes estudos. Em um estudo baseado em pesquisa internacional com 20.825 procedimentos entre 2003 e 2006, a taxa de mortalidade foi de 0,15\%. Outro achado do presente estudo foi um aumento crescente nas taxas de mortalidade precoces entre 2010 e 2015, que pode ser explicado por dois fatores importantes: primeiro, foram identificadas maiores taxas de comorbidades como ICC, doença coronariana, pulmonar e renal crônica, explicando, em parte, o crescimento de 4,8 a 7,8\% nos anos citados; segundo, o volume de ablação para FA bem como o número de instituições aumentaram nos anos recentes, consequentemente há mais procedimentos sendo realizados em centros de baixo volume, que estão associados a maiores taxas de complicação. Com relação à principal causa de readmissão, a septicemia, os autores não conseguiram obter dados para comprovar se foi relacionada à fístula atrioesofágica, pois não havia codificação específica no código internacional de doenças. Os autores concluem que, nessa coorte contemporânea, nacionalmente representativa, de mundo real, a mortalidade precoce após ablação para $\mathrm{FA}$ foi de 0,46\%, com a maior parte das mortes ocorrendo na readmissão em 30 dias após o procedimento. Complicações no procedimento inicial foram preditores independentes de mortalidade precoce. Sepse e ICC foram as principais causas de readmissão associadas à mortalidade, e a implementação de estratégias para reduzir complicações nos procedimentos, otimizar o manejo de ICC e reduzir infecções nosocomiais podem ajudar a reduzir a mortalidade precoce após ablação de FA.

*Cheng EP, Liu CF, Yeo I, Markowitz SM, Thomas G, Ip JE, et al. Risk of Mortality Following Catheter Ablation of Atrial Fibrillation. J Am Coll Cardiol. 2019;74(18):2254-64. https://doi.org/10.1016/j.jacc.2019.08.1036 